\title{
Nivolumab after sorafenib failure in liver recipients with hepatocellcular carcinoma recurrence
}

\author{
Ji Eun KWON, Manuel LIM, Jaehun YANG, Eun Sung JEONG, Kyeong Deok KIM, \\ Okjoo LEE, Jinsoo RHU, Gyu-Seong CHOI, Jong Man KIM*, Jae-Won JOH
}

Department of Surgery, Samsung Medical Center, Sungkyunkwan University School of Medicine, Seoul, Korea

Introduction: Several treatments have recently been known for hepatocellcular carcinoma (HCC) who have failed to treat sorafenib. Immune checkpoint inhibitors have proven to be effective, but data on the safety and efficacy of liver transplant (LT) patients are limited. We aimed to report the effect of nivolumab after failure of sorafenib treatment in patients with recurrent HCC after liver transplantation.

Methods: This study retrospectively evaluated patients who failed sorafenib for recurrent HCC treatment after liver transplantation in a single center from March 2007 to December 2018. Patients with recurrent hepatocellular carcinoma after liver transplantation who received regorafenib or nivolumab after sorafenib treatment failure were included.

Results: Of the 53 study patients, 8 received regorafenib and 10 received nivolumab. There is no difference in the characteristics of the two groups. Median overall survival (OS) from LT or HCC recurrence was 58.1 months and 40.2 months in the novolumab group and was 25.6 months and 17.6 months in the non-nivolumab group ( $p=0.104$ and $p=0.143$, respectively). The median OS from nivolumab use was 5.3 months and the median OS from sorafenib use in non-nivolumab group was 14.8 months $(p=0.657)$. All 10 patients receiving nivolumab failed to control HCC progression and 6 patients $(60 \%)$ were dead due to HCC progression during a median follow-up period of 2.4 months (interquartile range, 1.8-7.2 months) from the onset of nivolumab.

Conclusions: Present study suggests that the use of nivolumab in LT patients with recurrent HCC has not shown a clear function in inhibiting HCC progression yet. 